\title{
A Semi-automatic Endocardial Border Detection Method for 4D Ultrasound Data
}

\author{
Marijn van Stralen ${ }^{1}$, Johan G. Bosch ${ }^{1}$, Marco M. Voormolen ${ }^{2,3}$, Gerard van Burken ${ }^{1}$, \\ Boudewijn J. Krenning ${ }^{3}$, Charles T. Lancée ${ }^{3}$, Nico de Jong ${ }^{2,3}$, and Johan H.C. Reiber ${ }^{1,2}$ \\ ${ }^{1}$ Leiden University Medical Center, Radiology, Division of Image Processing C2-S, \\ P.O. Box 9600, 2300 RC Leiden, The Netherlands \\ \{M.van_Stralen, J.G.Bosch, G.van_Burken, J.H.C.Reiber\}@lumc.nl \\ ${ }^{2}$ ICIN, Interuniversity Cardiology Institute of the Netherlands, Utrecht, The Netherlands \\ ${ }^{3}$ Erasmus Medical Center, Thoraxcenter, Rotterdam, The Netherlands \\ \{M.Voormolen, B.Krenning, C.Lancee, N.deJong\} @erasmusmc.n1
}

\begin{abstract}
We propose a semi-automatic endocardial border detection method for $3 \mathrm{D}+\mathrm{T}$ cardiac ultrasound data based on pattern matching and dynamic programming, operating on $2 \mathrm{D}$ slices of the $3 \mathrm{D}+\mathrm{T}$ data, for the estimation of LV volume, with minimal user interaction. It shows good correlations with MRI ED and ES volumes ( $\mathrm{r}=0.938)$ and low interobserver variability $(y=1.005 x-16.7, r=0.943)$ over full-cycle volume estimations. It shows a high consistency in tracking the user-defined initial borders over space and time.
\end{abstract}

\section{Introduction}

For diagnosis of cardiovascular diseases, the volume and ejection fraction of the left heart chamber are important clinical parameters. 3D ultrasound offers good opportunities to visualize the whole left ventricle (LV) over the complete cardiac cycle. 3D ultrasound is non-invasive, relatively cheap, flexible in use and capable of accurate volume measurements. New, fast 3D ultrasound imaging devices are entering the market and have the potential of allowing such measurements rapidly, reliably and in a user-friendly way - provided that a suitable automated analysis is available. Manual segmentation of the large data sets is very cumbersome and suffers from inconsistencies and high variability. On the other hand, the human expert's interpretation and intervention in the detection is often essential for good results. Therefore a semi-automatic segmentation approach seems most suitable.

Some methods for segmentation of 4D echocardiographic images have been published. Angelini et al. [1] have reported on a wavelet-based approach for 4D echocardiographic image enhancement followed by an LV segmentation using standard snakes. Montagnat et al. [2] use a 2-simplex mesh and a feature detection based on a simple cylindrical gradient filter. Sanchez-Ortiz, Noble et al. [3] used multi-scale fuzzy clustering for a rough segmentation in 2D longitudinal slices. Bsplines are used for 3D surface fitting in each time frame. These methods have not been validated successfully on a reasonable data set. The most practical approach is described by Schreckenberg [4]. It uses active surfaces that are controlled by difference-of-boxes operators applied to averages and variances of the luminance. 
This technique is implemented in a commercially available workstation (4D LV Analysis, TomTec, Germany). The general experience is that this technique requires much initialization and corrections and a consistent segmentation is still hard to reach.

We present a new semi-automatic endocardial border detection method for time series of 3D cardiac ultrasound data. Our method is based on pattern matching and dynamic programming techniques and combines continuity, robustness and accuracy in $2 \mathrm{D}$ cross sections with the spatial and temporal continuity of the $4 \mathrm{D}$ data. It aims at optimally using a limited amount of user interaction (capturing essential information on shape and edge patterns according to the user's interpretation of the ultrasound data) to attain a fast, consistent and precise segmentation of the left ventricle.

Although the presented method is general and suitable for any apically acquired 3D set, we performed this study on a special type of image data acquired with a new device: the Fast Rotating Ultrasound (FRU-) transducer. The transducer has been developed by the Department of Experimental Echocardiography of the Erasmus MC, the Netherlands [5,6]. It contains a linear phased array transducer that is continuously rotated around its image-axis at very high speed, up to 480 rotations per minute (rpm), while acquiring 2D images. A typical data set is generated during 10 seconds at 360 rpm and 100 frames per second (fps). The images of the left ventricle are acquired with the transducer placed in apical position. The rotation axis will approach the LV long axis in the ideal situation. The analysis assumes that the rotation axis lies within the LV lumen and inside the mitral ring.

As a consequence of the very high continuous rotation speed, the images have a curved image plane (Fig. 1a). During the acquisition, the probe rotates about $22^{\circ}$ per image with the typical settings given above. The combination of these curved image planes, and the fact that the acquisition is not triggered by or synchronized to the ECG signal, results in an irregular distribution over the $3 \mathrm{D}+\mathrm{T}$ space. A single cardiac cycle in general is not sufficient for adequate coverage of the whole $3 \mathrm{D}+\mathrm{T}$ space; therefore, multiple consecutive heart cycles are merged. The cardiac phase for each image is computed offline using detected R-peaks in the ECG [7]. The total set of 2D images can be used for selection of a subset of images with a regular coverage of the $3 \mathrm{D}+\mathrm{T}$ space and/or for the generation of a 4D voxel set. We perform analysis on the prior.

\section{Methods}

\subsection{Frame Selection}

To achieve adequate coverage of the whole 3D+T space, multiple consecutive cardiac cycles are merged and an optimal subset $S$ of the total set of frames $T$ is selected. This subset is an optimal fit of the frames on a chosen $A * P$ matrix of $A$ equidistant rotation angles and $P$ cardiac phases, minimizing the total deviation in rotation angle and cardiac phase. Moreover, the variation in acquisition time over the subset is minimized to limit possible motion artifacts. The constraints are translated into the following cost functions that will be minimized over the total subset $S$, 


$$
\begin{gathered}
S=\underset{T}{\arg \min } \sum_{i=1}^{A} \sum_{j=1}^{P}\left(\underset{b \in C_{i, j}}{\arg \min }\left(c_{\text {angle }}\left(\alpha_{b}, i\right)+c_{\text {phase }}\left(p_{b}, j\right)+c_{\text {time }}\left(t_{b}\right)\right)\right) \text {, where } \\
c_{\text {angle }}(\alpha, i)=k_{l}\left|\alpha_{\text {target }}(i)-\alpha\right| ; c_{\text {phase }}(p, j)=k_{2}\left|p_{\text {target }}(j)-p\right| ; c_{\text {time }}(t)=k_{3}\left|t_{S}-t\right| .
\end{gathered}
$$

$C_{i, j}$ is the set of candidate images for angle $\# i$ and phase $\# j . c_{\text {angle }}$ and $c_{\text {phase }}$ describe the costs of selecting an image $b$ with angle $\alpha_{b}$ and phase $p_{b}$ for a chosen $\alpha_{\text {target }}$ and $p_{\text {target }}$. $k_{1}, k_{2}$ and $k_{3}$ are weighting coefficients (typically equal). Since the cost $c_{\text {time }}$ is dependent on $t_{S}$ (the average acquisition time of the subset itself), the minimization of the costs of set $S$ is achieved in an iterative manner.

\subsection{Border Detection Approach}

We base our method on the knowledge that the edge patterns of the endocardial border can be complex, very different from patient to patient and even between regions within an image set. The border position need not correspond to a strong edge and may be only definable from 'circumstantial evidence' as identified by an expert observer. Rather than applying artificial, idealized edge models or templates derived from a large training set, we propose a tracking approach based on edge templates extracted from the user-defined initial borders in the patient's own images.

The method is based on these continuity assumptions (in order of strength):

1. border continuity in separate $2 \mathrm{D}$ slices of the left ventricle;

2. spatial continuity of shape and gray value edge patterns over the LV surface in 3D;

3. temporal and cyclic motion continuity of the endocardium.

For the FRU transducer, within the original 2D images, both spatial and temporal distances between neighboring samples are smaller than towards adjacent images in angle and phase; therefore, border continuity is supposed to be strongest here. For other devices, similar considerations apply.

The method is initialized from four manually drawn contours, taken from two roughly perpendicular views (more or less corresponding to two- and four-chamber cross sections) in two phases: end-diastole (ED) and end-systole (ES). These are used to initialize a model for the edge patterns near the 3D LV surface over time and a 3D shape model of the LV endocardial surface over the entire heart cycle. Both models are inherently 4-dimensional and can be polled at any spatial position and phase.

The actual border detection takes place in individual 2D images from the selected subset and is an extension of an approach for $2 \mathrm{D}+\mathrm{T}$ sequences earlier developed by Bosch et al. [8]. For each image $b \in S$ (of phase $p_{b}$ and angle $\alpha_{b}$ ), an estimation of the border shape is derived by intersecting the 3D shape model at phase $p_{b}$ by the (curved) image plane for angle $\alpha_{b}$. The edge templates are also interpolated for the desired $p_{b}$ and $\alpha_{b}$. In the 2D image, a neighborhood of the estimated shape is resampled along lines perpendicular to the shape estimate. Using a template matching with the local edge templates, the similarity of each candidate edge point to the template is calculated. Dynamic programming is applied to find an optimal continuous border within the restrictions posed by the $3 \mathrm{D}$ model. In this way, the $4 \mathrm{D}$ surface and edge pattern models guard the (looser) spatial and temporal consistency of the detection, while the dynamic programming approach supplies a continuous and optimal 


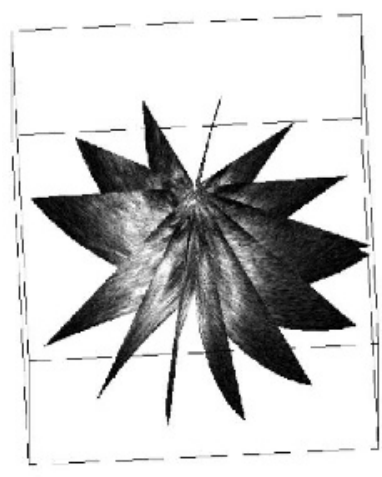

A

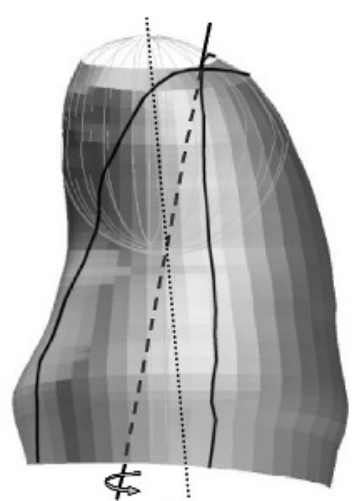

B

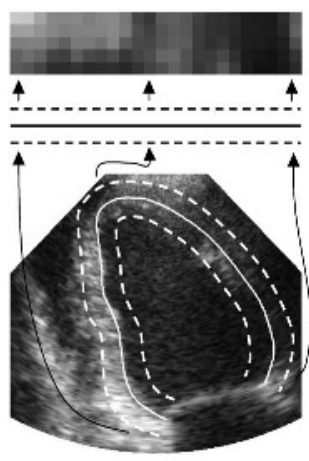

C

Fig. 1. (a) A sequence of seven consecutive FRU images with curved image planes. (b) 3D surface model. The LAX estimate (dotted) and the rotation axis (dashed) are shown, together with the reconstruction of the apex by spline interpolation (light gray) from two manually drawn contours (solid black). (c) The extraction of a stylized edge pattern from the image

detection locally. The set of detected contours describes the 3D endocardial surface over the whole cardiac cycle from which LV volumes, ejection fraction etc. can be computed.

The method is suitable for effective correction of the detection in other 2D images (although this was not yet used or evaluated in this study). A corrected contour will be treated as an additional manual contour and a complete new set of shape and edge pattern models will be interpolated, and all images are redetected. In this manner, corrections will cumulatively lead to a superior global solution.

\subsection{D Surface Models}

As said, for two cardiac phases (ED and ES) a 3D surface model of the LV endocardium is constructed from two almost perpendicular contours. During the acquisition the rotation axis is more or less aligned with the long axis (LAX) of the left ventricle, but in practice there may be a considerable mismatch (Fig. 1b). This implies that the two image planes do not contain the true apex of the heart, and estimating the position and shape of the true apex (and the LV long axis) is a nontrivial issue. The local long axes in the 2D manually drawn contours are defined as the lines between the midpoint of the mitral valve (MV) and the 2D apex. We estimate the 3D LV long axis from the local long axes by computing the intersection of the planes perpendicular to these images through the local long axis in the image.

The endocardial surface is estimated by expressing the two contours in a cylindrical coordinate system with respect to the estimated LV long axis and applying a Kochanek spline interpolation [9] of the radial component over the angle in planes perpendicular to the long axis. This is a natural approximation of the ellipsoidal shape of the left ventricle. Since the two image planes generally do not intersect the real apex, the apical cap of the LV surface cannot be estimated simply from the two 
manually drawn contours, as shown in Fig. 1b. Therefore, near the 3D apex we use a spherical coordinate system oriented around the LV long axis, centered at $3 / 4^{\text {th }}$ of its length. The surface is estimated by a Kochanek spline interpolation of the radial component over the elevation angle for multiple rotation angles.

A contour estimate for any image at a given rotation angle and cardiac phase can be made by intersecting its curved image plane with the $3 \mathrm{D}$ contour models in ED and ES and then linearly interpolating between the two resulting ' $2 \mathrm{D}$ ' contours over cardiac phase to get the contour estimate at the desired cardiac phase.

\subsection{Edge Pattern Model}

The desired edges are tracked over space and time by applying a pattern matching approach with edge templates. These edge patterns are derived from the manually drawn contours and interpolated over the (phase, angle) space.

The image is resampled clockwise along the manually drawn contour, on line segments perpendicular to this contour from the inside out. The gray values on these line segments are smoothed and subsampled to form a stylized edge pattern for this contour (Fig. 1c). A typical edge pattern for a single 2D frame is represented by 32 positions along the contour and 5 samples around each edge position.

A linear interpolation over cardiac phase is performed between the edge patterns in ED and ES. The interpolation over rotation angle is less straightforward. Since the character of the edge pattern is strongly related to the angle between the endocardial border and the ultrasound beam and the distance from the transducer, the pattern changes considerably over the rotation angle, especially when the angle between the rotation axis and LV long axis is substantial. For images with rotation angles opposite $\left(\bullet 180^{\circ}\right)$ to those with the manually drawn contours, the image appears nearly mirrored and the mirrored (anticlockwise) edge pattern is used. For angles in between, the edge patterns are linearly interpolated.

\subsection{Contour Detection}

With an edge pattern and initial contour for each image $b \in S$ (of phase $p_{b}$ and angle $\alpha_{b}$ ), we can now detect the individual endocardial borders. In a neighborhood of the initial contour, the image is resampled into an $N^{*} M$ rectangular array by sampling $N$ points along $M$ scan lines perpendicular to the shape. From the stylized edge pattern for $\left(p_{b}, \alpha_{b}\right)$ an edge template for each scan line is extracted. For all nodes in the array, the sum of absolute differences with its respective edge template defines the cost of the node. We now use a straightforward dynamic programming approach [10] to find the optimal connective path through the array. A connective path contains exactly one node per line and the positions on consecutive lines cannot differ more than a predefined side step size. Dynamic programming is a well-known technique [10] that finds the optimal path (the path with the lowest sum of costs) out of all possible connective paths in an effective manner by calculating lowest cumulative costs for consecutive layers (lines) while keeping track of the partial optimal paths. Backtracking from the node with lowest cumulative cost in the last layer delivers the 


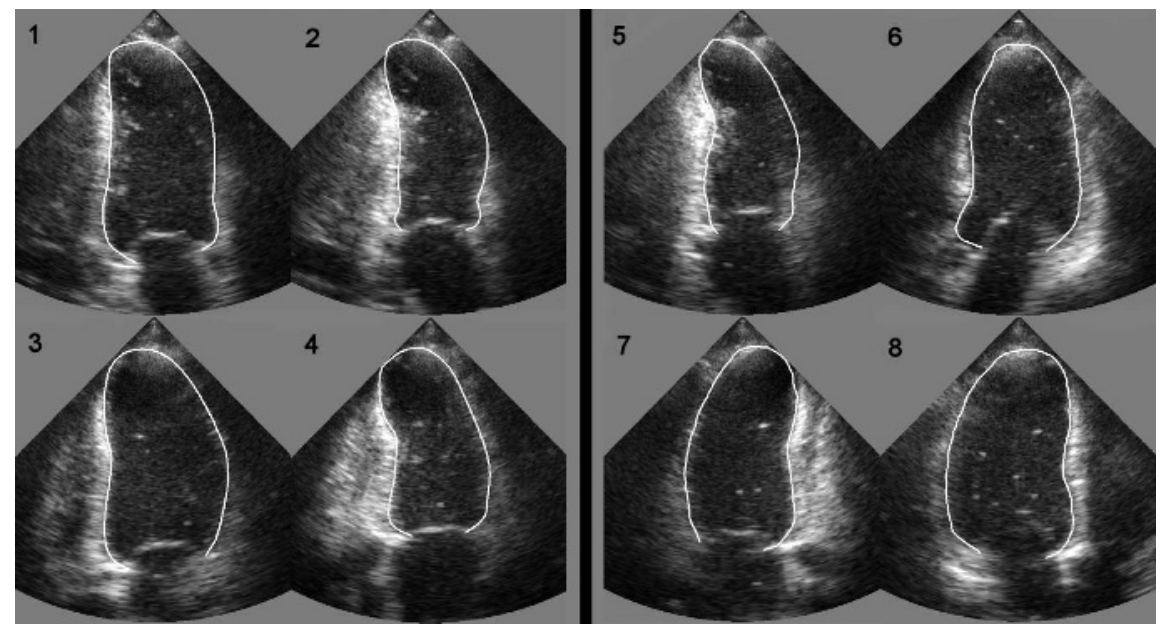

Fig. 2. Detection examples: frames at different (phase\#, angle\#) with contours. Left: 4 frames with manual contours, resp. 1: ED 2c (1,1), 2: ES 2c (6,1), 3: ED 4c (1,3), 4: ES 4c (6,3). Right: 4 frames with detected contours, resp. 5: frame $(8,2), 6:(14,5), 7:(4,8), 8:(14,9)$

overall optimal path. Smoothness constraints are enforced by applying additive costs for sidestepping during cumulative cost calculation. To limit the influence of lines with relatively poor image information, this additive penalty is calculated per line from the statistics of node costs per line with respect to overall cost statistics, such that relatively unreliable lines get higher penalties for sidestepping.

For each phase $p_{i}$, the detected contours of all angles $\alpha_{i}$ together constitute a 3D mesh that describes the endocardial surface. We observe the volume of the left ventricle over the whole cardiac cycle, by calculating the volumes inside the surface meshes of all selected heart phases.

\section{Results}

We performed a preliminary validation study for this method on a group of 14 subjects with different diagnoses of cardiovascular disease. MRI ED and ES volumes on these patients were determined in parallel with the 3D US study. For all patients, subsets of images were created with $P=16$ phases and $A=10$ angles. After establishing equivalent tracing conventions, two observers individually analyzed all subsets.

Reading and converting the data and the automated selection of the subset took 7 minutes per patient on average. After the drawing of the four contours, the fully automated detection of the other 156 contours took approximately 90 seconds per patient. No contour corrections were allowed afterwards. Some examples of manual and detected contours are shown in Fig. 2. From the analyses of both observers, interobserver variabilities of $\mathrm{ED} / \mathrm{ES}$ volumes, $\mathrm{EF}$ and all other volumes were determined, as well as averages that were correlated to MRI ED and ES volumes. 

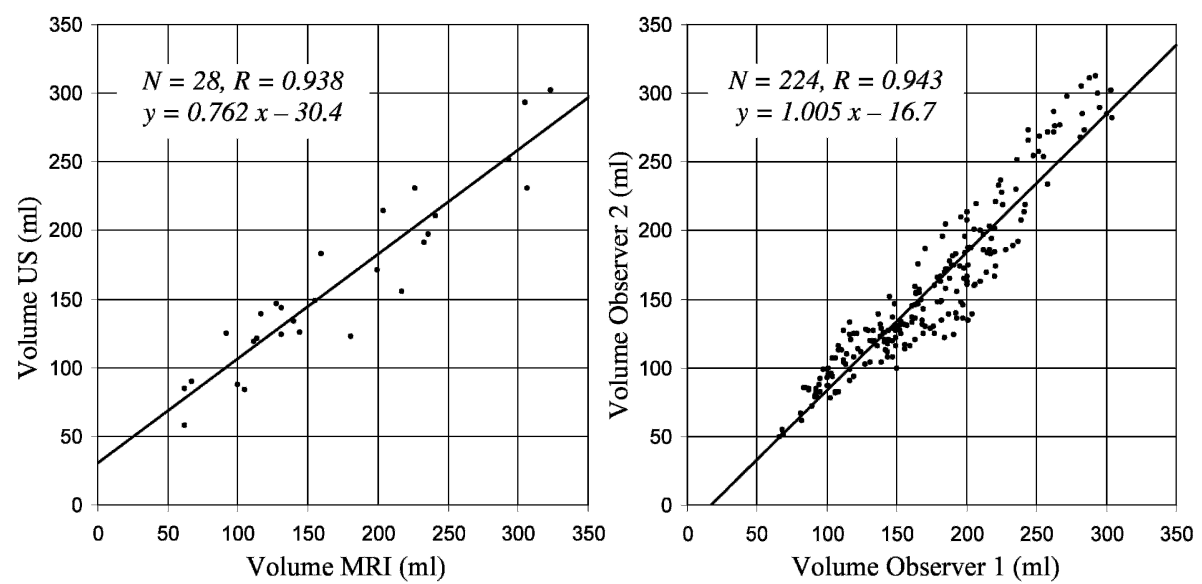

Fig. 3. (L) ED/ES volumes US vs. MRI. (R) Interobserver variability of full-cycle volumes

Results of 3DUS (average of the two observers) vs. MRI are shown in Table 1 and Fig. 3a. These show the ED and ES volumes and the corresponding correlation coefficients and regression equations.

A high correlation of $\mathrm{r}=0.938$ was found between MRI and US volumes. Overall the MRI volumes were slightly higher. This has been reported in many studies and can be attributed to different tracing conventions. The differences between MRI and US volumes were not significant (paired t-test, $\mathrm{p}=0.068$ ). EF results showed a reasonable difference of $3.3+/-8.6 \%$ (regression $y=0.493 x+16.6 \%, r=0.755$ ).

For the interobserver variability, results are presented in Table 1 (ED and ES) and Fig. 3b (full-cycle). For ED and ES volumes only, the differences were $15.0+/-21.0$ (av +/- SD) with a regression of $y=1.013 x-17.4(\mathrm{r}=0.949)$. We found similar differences of $15.6+/-19.3$ over all other phases $(\mathrm{y}=1.003 \mathrm{x}-16.1, \mathrm{r}=0.943)$. This difference (ED/ES vs. other phases) was not significant (unpaired t-test, $\mathrm{p}=0.628$ ). This implies that the detection does not introduce additional variability/errors with respect to interobserver differences from the manual contours.

Absolute differences in volumes may seem high, but this is partially due to the dilated hearts in the set and the consequent high average volumes (average MRI ED $=209$ $\mathrm{ml}$ ). The systematic differences in tracing between observers were equally reflected in the detected contours, which is encouraging.

Table 1. ED/ES volumes and correlations of 3DUS vs. MRI and Observer 1 vs. Observer 2

\begin{tabular}{c|cccccc} 
& \multicolumn{2}{|c}{ ED Volume $(\mathbf{m l})$} & \multicolumn{2}{c}{ ES Volume $(\mathbf{m l})$} & \multirow{2}{*}{ Correlation } & \multirow{2}{*}{ Regression } \\
\hline Average & SD & Average & SD & & \multirow{2}{*}{$0.762 \mathrm{x}+$} \\
URI & 209 & 67 & 133 & 69 & \multirow{2}{*}{0.938} & 30.4 \\
\hline Obs.1 & 194 & 57 & 127 & 50 & & $1.013 \mathrm{x}-$ \\
Obs.2 & 203 & 56 & 133 & 48 & \multirow{2}{*}{0.949} & 17.4
\end{tabular}




\section{Conclusions and Future Work}

We presented a new semi-automatic endocardial border detection method for $4 \mathrm{D}$ ultrasound data. This method offers fast and reasonably precise automated border detection with minimal user interaction and very promising results. However, one should bear in mind that this analysis is only preliminary. Methods have not yet been optimized and smaller flaws still exist. In some cases phase information was inexact, hampering the model interpolations. Also, the linear model interpolations currently disregard the complex shape and motion of the mitral valve, which is known to move nonlinearly in diastole. In addition, some distinct local misdetections were found that will be addressed in future research. Furthermore, the method was designed with advanced correction features in mind (see 2.2). These have not yet been used or tested in this analysis. It is to be expected that this will allow significantly better results with little interaction; similar improvements were found with its predecessor for the $2 \mathrm{D}+\mathrm{T}$ analyses.

Despite the fact that this method is optimized for data of the FRU-transducer, the algorithm can be easily adapted to data of other image acquisition systems, for example 4D voxel sets. The detection will then be performed in 2D slices through the LV long axis.

\section{References}

1. Angelini, E.D. et al., LV Volume Quantification via Spatiotemporal Analysis of RealTime 3D Echocardiography, IEEE Trans Medical Imaging 20 (2001), pp. 457-469, 2001

2. Montagnat, J., Delingette, H., Space and Time Shape Constrained Deformable Surfaces for 4D Medical Image Segmentation. MICCAI 2000, Springer, vol. LNCS 1935, pp. 196-205

3. Sanchez-Ortiz, G.I. et al., Automating 3D Echocardiographic Image Analysis, MICCAI 2000, Springer, LNCS 1935, pp. 687-696

4. Schreckenberg, M. et al., Automatische Objecterkennung in 3D-echokardiographiesequenz-en auf Basis Aktiver Oberflächenmodellen und Modellgekoppelter Merkmalsextraktion, In: Lehman, T. et al. (eds.): Bildverarbeitung für die Medizin, Springer, V087, 1998

5. Djoa, K.K. et al., A Fast Rotating Scanning Unit for Real-Time Three Dimensional Echo Data Acquisition, Ultrasound in Med. \& Biol., vol 26(5), pp. 863-869, 2000

6. Voormolen, M.M. et al., A New Transducer for 3D Harmonic Imaging, Proc. Of IEEE Ultrasonics Symposium, pp. 1261-1264, 2002

7. Engelse, W.A.H., Zeelenberg, C., A Single Scan Algorithm for QRS-detection and Feature Extraction, Computers in cardiology 6, pp. 37-42, 1979

8. Bosch, J.G. et al., Overview of Automated Quantitation Techniques in 2D Echocardiography, In: Reiber J.H.C. and Van der Wall, E.E. (eds.): What's New in Cardiovascular Imaging, edited by, Kluwer academic publishers, pp. 363-376, 1998

9. Kochanek, D., Bartels, R., Interpolating Splines with Local Tension, Continuity, and Bias Control, Computer Graphics, vol. 18, no. 3, pp. 33-41, 1984.

10. Sonka, M. et al., Segmentation, Ch. 5 of Image Processing, Analysis and Machine Vision, PWS Publishing, pp. 158-163, 1999 